TRANSACTIONS OF THE

AMERICAN MATHEMATICAL SOCIETY

Volume 348, Number 10, October 1996

\title{
SINGLE GENERATOR PROBLEM
}

\author{
JUN-ICHI TANAKA
}

Dedicated to Professor Yuji Ito on his 60th birthday

\begin{abstract}
Using the Stone-Čech compactification $\beta \mathbf{Z}$ of integers, we introduce a free extension of an almost periodic flow. Together with some properties of outer functions, we see that, in a certain class of ergodic Hardy spaces $H^{p}(\mu)$, $1 \leq p \leq \infty$, the corresponding subspaces $H_{0}^{p}(\mu)$ are all singly generated. This shows the existence of maximal weak-* Dirichlet algebras, different from $H^{\infty}$ of the disc, for which the single generator problem is settled.
\end{abstract}

\section{INTRODUCTION}

Let $\Omega$ be a compact Hausdorff space on which the real line $\mathbf{R}$ acts as a topological transformation group. This means that there is a one-parameter group $\left\{U_{t}\right\}_{t \in \mathbf{R}}$ of homeomorphisms of $\Omega$ such that $(\omega, t) \rightarrow U_{t} \omega$ is continuous on $\Omega \times \mathbf{R}$. The pair $\left(\Omega,\left\{U_{t}\right\}_{t \in \mathbf{R}}\right)$ is referred to as a (continuous) flow and the translate $U_{t} \omega$ of $\omega$ by $t$ is denoted by $\omega+t$. In most of our discussion, each flow $\left(\Omega,\left\{U_{t}\right\}_{t \in \mathbf{R}}\right)$ is free in the sense that if $U_{t} \omega=\omega$ for some $\omega$ in $\Omega$, then $t=0$. Let $C(\Omega)$ be the space of all continuous complex-valued functions on $\Omega$. Then the dual space of $C(\Omega)$ is the space of all bounded regular Borel measures on $\Omega$, which is denoted by $M(\Omega)$. Recall that an invariant measure in $M(\Omega)$ is said to be ergodic if each invariant measurable set is either negligible or has negligible complement.

We denote by $L^{p}(\mathbf{R})$ and $H^{p}(\mathbf{R}), 1 \leq p \leq \infty$, the usual Lebesgue and Hardy spaces on $\mathbf{R}$, respectively. A function $\phi$ in $C(\Omega)$ is analytic, if $t \rightarrow \phi(\omega+t)$ lies in $H^{\infty}(\mathbf{R})$ for each $\omega$ in $\Omega$. We denote by $A(\Omega)$ the space of all analytic functions in $C(\Omega)$. Then $A(\Omega)$ is a uniformly closed subalgebra of $C(\Omega)$ that contains constants. If $\left(\Omega,\left\{U_{t}\right\}_{t \in \mathbf{R}}\right)$ is free, then $A(\Omega)$ separates the points on $\Omega$, and $A(\Omega)$ is a uniform algebra ([14, Theorem 1]). Let $\mu$ be an invariant, ergodic, probability measure in $M(\Omega)$. Then $\mu$ is a representing measure for $A(\Omega)$, and $A(\Omega)$ is a weak- ${ }^{*}$ Dirichlet algebra in $L^{\infty}(\mu)\left([13\right.$, Theorem I] $)$. The Hardy space $H^{p}(\mu), 1 \leq p<\infty$, denotes the closure of $A(\Omega)$ in $L^{p}(\mu)$, while $H^{\infty}(\mu)$ is the weak-* closure of $A(\Omega)$ in $L^{\infty}(\mu)$. Let $H_{0}^{p}(\mu)$ be the space of all functions $\phi$ in $H^{p}(\mu)$ such that $\int_{\Omega} \phi d \mu=0$. The ideal $A_{0}(\Omega)$ of $A(\Omega)$ is similarly defined. A closed subspace $\mathbf{M}$ of $L^{p}(\mu)$ is invariant if $A(\Omega) \cdot \mathbf{M}$ is contained in $\mathbf{M}$. When $C(\Omega) \cdot \mathbf{M}$ is not contained in $\mathbf{M}$, it is said to be simply invariant. Of course, $H^{p}(\mu)$ and $H_{0}^{p}(\mu)$ are simply invariant subspaces. For

Received by the editors February 3, 1994 and, in revised form, May 24, 1995.

1991 Mathematics Subject Classification. Primary 46J10, 46J15, 43A17; Secondary 30H05, $54 \mathrm{H} 20$.

Key words and phrases. Ergodic Hardy spaces, outer functions, single generators, cocycles.

This research was partially supported by Grants 03640174, 04640183 and 05640221 from the Japanese Ministry of Education. 
a function $\phi$ in $L^{p}(\mu), \mathbf{M}[\phi]$ denotes the smallest invariant subspace that contains the function $\phi$. It is known that $\mathbf{M}[\phi]$ is simply invariant if and only if

$$
\rho(\phi)(\omega):=\int_{-\infty}^{\infty} \log |\phi(\omega+t)| \frac{d t}{1+t^{2}}>-\infty, \quad \mu \text {-a.e. } \omega \in \Omega,
$$

(see [9, Theorem 22]). A simply invariant subspace $\mathbf{M}$ is said to be singly generated if $\mathbf{M}=\mathbf{M}[\phi]$ for some $\phi$ in $\mathbf{M}$, which is called a single generator of $\mathbf{M}$.

Recall that a function of modulus one is called a unitary function and an analytic unitary function is said to be inner. A function $\phi$ in $H^{p}(\mu)$ is outer if it satisfies the inequality

$$
\log \left|\int_{\Omega} \phi d \mu\right|=\int_{\Omega} \log |\phi| d \mu>-\infty .
$$

Then $\phi$ is a single generator of $H^{p}(\mu)$ if and only if $\phi$ is outer in $H^{p}(\mu)$. If the flow is periodic, then $H_{0}^{p}(\mu)$ is also singly generated. This is because such a flow is conjugate to rotation on the circle and, therefore, the associated Hardy spaces are the classical ones. However, if the flow is not periodic (and the measure is ergodic) it has been unknown, until now, whether $H_{0}^{p}(\mu)$ can be singly generated. In the literature, this has come to be known as the single generator problem (see [5, Remark, p. 158], [6, Chapter VII, §7], and [9, §5.4]). The purpose of this note is to exhibit a method for constructing a free flow $\left(\Omega,\left\{U_{t}\right\}_{t \in \mathbf{R}}\right)$ together with an invariant ergodic probability measure $\mu$ such that the subspace $H_{0}^{p}(\mu)$ is singly generated, $1 \leq p \leq \infty$. Our result thus settles, in a certain sense, the single generator problem, but begs the question of whether every properly ergodic flow has the property that the associated $H_{0}^{p}(\mu)$-spaces are singly generated.

We refer the reader to [4], [6, Chapter VII], and [9] for further details of analyticity on flows and to [7] for some results about classical Hardy spaces which we shall use.

In order to state our theorem, we need more notation and definitions. Let $\Gamma$ be a dense subgroup of $\mathbf{R}$, endowed with the discrete topology, and let $K$ be the dual group of $\Gamma$. For each $t$ in $\mathbf{R}, e_{t}$ denotes the element of $K$ defined by $e_{t}(\lambda)=e^{i \lambda t}$ for $\lambda$ in $\Gamma$. Then the map $t \rightarrow e_{t}$ embeds $\mathbf{R}$ continuously onto a dense subgroup of $K$. Let us define

$$
T_{t} x=x+e_{t}, \quad x \in K,
$$

for each $t$ in $\mathbf{R}$. Then the flow $\left(K,\left\{T_{t}\right\}_{t \in \mathbf{R}}\right)$ is strictly ergodic, meaning that there is a unique invariant probability measure in $M(K)$, which is the normalized Haar measure $\sigma$ on $K$. In this situation, a function $\phi$ in $L^{p}(\sigma)$ lies in $H^{p}(\sigma)$ if and only if its Fourier coefficients,

$$
a_{\lambda}(\phi)=\int_{K} \overline{\chi_{\lambda}(x)} \phi(x) d \sigma(x),
$$

vanish for negative $\lambda$ in $\Gamma$, where $\chi_{\lambda}$ denotes the character on $K$ defined by $\chi_{\lambda}(x)=$ $x(\lambda)$.

The single generator problem was originally formulated in the context of $H_{0}^{2}(\sigma)$ by $\mathrm{H}$. Helson. Although we are unable to decide this particular case of the problem, we shall see that our main result is very intimately related to it. 
Recall that a flow $\left(\Sigma,\left\{S_{t}\right\}_{t \in \mathbf{R}}\right)$ is an extension of $\left(K,\left\{T_{t}\right\}_{t \in \mathbf{R}}\right)$ if there is a continuous map $\pi$ of $\Sigma$ onto $K$ such that

$$
\pi \circ S_{t}(\omega)=T_{t} \circ \pi(\omega), \quad \omega \in \Sigma,
$$

for all $t$ in $\mathbf{R}$. Then $\left(K,\left\{T_{t}\right\}_{t \in \mathbf{R}}\right)$ is also called a factor of $\left(\Sigma,\left\{S_{t}\right\}_{t \in \mathbf{R}}\right)$. If, in particular, $\pi$ is a homeomorphism of $\Sigma$ onto $K$, we say that the flows are conjugate. Define the isometric isomorphism of $C(K)$ into $C(\Sigma)$ by

$$
\Pi(\phi)(\omega)=\phi \circ \pi(\omega), \quad \omega \in \Sigma .
$$

From (1.3), it follows that $\Pi$ maps $A(K)$ into $A(\Sigma)$.

Theorem 1.1. Let $\Gamma,\left(K,\left\{T_{t}\right\}_{t \in \mathbf{R}}\right)$, and $\sigma$ be as above, and let $1 \leq p \leq \infty$. Then there is a free extension $\left(\Sigma,\left\{S_{t}\right\}_{t \in \mathbf{R}}\right)$ of $\left(K,\left\{T_{t}\right\}_{t \in \mathbf{R}}\right)$ under the map $\pi$ of $\Sigma$ onto $K$, which satisfies the following properties:

(i) There is an invariant, ergodic, probability measure $\mu$ in $M(\Sigma)$ for which $H_{0}^{p}(\mu)$ has a single generator.

(ii) If $\Pi$ is the isomorphism of $C(K)$ into $C(\Sigma)$ defined by (1.4), then $\Pi$ extends to an isometry of $L^{p}(\sigma)$ into $L^{p}(\mu)$ that maps $H_{0}^{p}(\sigma)$ and $H^{p}(\sigma)$ to $H_{0}^{p}(\mu)$ and $H^{p}(\mu)$, respectively.

(iii) If $\Gamma$ is countable, then $\Sigma$ is metrizable.

We note that (ii) enables us to regard $H^{p}(\sigma)$ as a closed subspace of $H^{p}(\mu)$ and that the assumption of (iii) is equivalent to assuming that $K$ is metrizable.

In the next section, we establish some notation and elementary facts which we shall need. We develop in Section 3 a representation of almost periodic functions on $\mathbf{R}$. After preparing one lemma, the proof of Theorem 1.1 is given in Section 4 . In Section 5, we discuss some relations between Helson's work and our result. We close with several remarks in Section 6 .

The author would like to express his sincere gratitude to the referee for his valuable suggestions which improved the first version of this paper.

\section{AnALYTiCity ON FLOWS}

Let $\left(\Omega,\left\{U_{t}\right\}_{t \in \mathbf{R}}\right)$ be a flow, and let $f$ be a function in $L^{1}(\mathbf{R})$. Suppose that a Borel function $\phi$ on $\Omega$ satisfies that $t \rightarrow \phi(\omega-t) f(t)$ lies in $L^{1}(\mathbf{R})$ for each $\omega$ in $\Omega$. Then the convolution $\phi * f$ is defined by the Borel function

$$
\phi * f(\omega)=\int_{-\infty}^{\infty} \phi(\omega-t) f(t) d t, \quad \omega \in \Omega .
$$

Let $\mu$ be an invariant, ergodic, probability measure in $M(\Omega)$. When $\phi$ lies in $L^{1}(\mu), \phi * f$ is always defined by Fubini's theorem. Moreover, $\phi * f$ may be considered as a Bochner integral in $L^{1}(\mu)$. Observe that a function $\phi$ in $L^{p}(\mu)$ lies in $H^{p}(\mu)$ if and only if $\phi * \tilde{f}=0$ for all $f$ in $H^{1}(\mathbf{R})$, where $\tilde{f}(t)=f(-t)$. Let $P_{i r}(t), r>0$, be the Poisson kernel for the upper half-plane $\mathbf{R}_{+}^{2}$; explicitly, $P_{i r}(t)=r / \pi\left(t^{2}+r^{2}\right)$. We note that (1.1) may be rewritten as $\rho(\phi)(\omega)=(\log |\phi|) * P(\omega)>-\infty$, since $P_{i}(t)$ is an even function.

We denote by $H^{\infty}\left(\mathbf{R}_{+}^{2}\right)$ the space of all bounded analytic functions on $\mathbf{R}_{+}^{2}$. By Fatou's theorem, we may identify $H^{\infty}(\mathbf{R})$ with $H^{\infty}\left(\mathbf{R}_{+}^{2}\right)$. Let $\phi$ be a function in $H^{\infty}(\mu)$. For $z=u+i r$ in $\mathbf{R}_{+}^{2}$, we write

$$
\phi(\omega+z)=\phi(\omega+(u+i r))=\phi * P_{i r}(\omega+u), \quad \omega \in \Omega .
$$


Then the function $z \rightarrow \phi(\omega+z)$ lies in $H^{\infty}\left(\mathbf{R}_{+}^{2}\right)$ for $\mu$-a.e. $\omega \in \Omega$. We denote by $\mathcal{B}(\Omega)$ the $\sigma$-algebra of all Borel subsets of $\Omega$. Let $\left(K,\left\{T_{t}\right\}_{t \in \mathbf{R}}\right)$ and $\sigma$ be as in Section 1. Suppose that $\left(\Omega,\left\{U_{t}\right\}_{t \in \mathbf{R}}\right)$ is an extension of $\left(K,\left\{T_{t}\right\}_{t \in \mathbf{R}}\right)$ under the map $\pi$ of $\Omega$ onto $K$. Then if we set

$$
\pi^{-1}(\mathcal{B}(K))=\left\{\pi^{-1}(E) ; E \in \mathcal{B}(K)\right\}
$$

then $\pi^{-1}(\mathcal{B}(K))$ is a $\sigma$-subalgebra of $\mathcal{B}(\Omega)$, and $\sigma$ induces an invariant measure $\tilde{\sigma}$ on $\pi^{-1}(\mathcal{B}(K))$.

Let $\widetilde{\mathcal{B}}(K)$ be a $\sigma$-subalgebra of $\mathcal{B}(\Omega)$ containing $\pi^{-1}(\mathcal{B}(K))$ which is invariant under the translation by $\left\{U_{t}\right\}_{t \in \mathbf{R}}$. Suppose that $\tilde{\sigma}$ extends to $\widetilde{\mathcal{B}}(K)$, that is, two measure algebras $\left\langle\pi^{-1}(\mathcal{B}(K)), \tilde{\sigma}\right\rangle$ and $\langle\widetilde{\mathcal{B}}(K), \tilde{\sigma}\rangle$ are isomorphic in the sense of Boolean algebras. Then $L^{\infty}(\sigma)$ is identified with $L^{\infty}(\Omega, \widetilde{\mathcal{B}}(K), \tilde{\sigma})$, naturally. Let $\theta$ be a function in $L^{\infty}(\sigma)$. Then $\theta \circ \pi$ is said to lie in $C(\Omega)$ if $\theta \circ \pi$ differs from a function in $C(\Omega)$ by a $\tilde{\sigma}$-null function in $L^{\infty}(\Omega, \widetilde{\mathcal{B}}(K), \tilde{\sigma})$.

We next define

$$
\left\{\begin{array}{l}
\widetilde{C}(K)=\{\phi \circ \pi ; \phi \in C(K)\}, \\
\widetilde{A}(K)=\{\phi \circ \pi ; \phi \in A(K)\} .
\end{array}\right.
$$

Then $\widetilde{C}(K)$ is a selfadjoint closed subalgebra of $C(\Omega)$, which is translation-invariant, meaning that, if $\psi$ is in $\widetilde{C}(K)$, then the translation,

$$
U_{t} \psi(\omega)=\psi(\omega+t),
$$

lies in $\widetilde{C}(K)$ again for each $t$ in $\mathbf{R}$. Similarly, $\widetilde{A}(K)$ is also a translation-invariant closed subalgebra of $A(\Omega)$, although $\widetilde{A}(K)$ may not separate the points of $\Omega$. As we shall see later, if the topology on $\Omega$ is appropriately strong, then there appear functions $\theta$ in $L^{\infty}(\sigma) \backslash C(K)$ for which the functions $\theta \circ \pi$ lie in $C(\Omega)$.

It follows from the Riesz representation theorem and the Hahn-Banach theorem that $\tilde{\sigma}$ may be extended to a probability measure in $M(\Omega)$. In the following lemma, we choose a suitable extension.

Lemma 2.1. Under the above assumptions, let $\theta$ be a nonnegative function in $L^{\infty}(\sigma)$ such that $\theta \circ \pi$ lies in $C(\Omega)$. Suppose that $\int_{K} \theta d \sigma>0$. Then there is an invariant, ergodic, probability measure $\mu$ in $M(\Omega)$ for which

$$
\int_{\Omega} \theta \circ \pi d \mu>0
$$

Proof. Since $\left(K,\left\{T_{t}\right\}_{t \in \mathbf{R}}\right)$ is strictly ergodic, the equation,

$$
\int_{K} \phi d \sigma=\int_{\Omega} \phi \circ \pi d \nu
$$

holds on $C(K)$, for any invariant probability measure $\nu$ in $M(\Omega)$.

We now show that, by choosing a suitable $\nu,(2.3)$ holds on $C(K) \cup\{\theta\}$. Let $\mathbf{S}$ be the subspace of $C(\Omega)$ generated by $\widetilde{C}(K)$ and $\left\{U_{t} \theta \circ \pi ; t \in \mathbf{R}\right\}$. Since $\theta$ is in $L^{\infty}(\sigma), U_{t} \theta \circ \pi$ lies in $L^{\infty}(\Omega, \widetilde{\mathcal{B}}(K), \tilde{\sigma})$. Then we may define the positive linear functional on $\mathbf{S}$ by

$$
\Upsilon(\psi)=\int_{\Omega} \psi d \tilde{\sigma}, \quad \psi \in \mathbf{S} .
$$

We remark that the inequality

$$
\|\psi\|_{\infty} \geq \inf \{s ;|\psi(\omega)| \leq s \quad \tilde{\sigma} \text {-a.e. } \omega \in \Omega\}
$$


holds. From the Hahn-Banach theorem, there is a probability measure $\lambda$ in $M(\Omega)$ which represents a positive extension of $\Upsilon$ to $C(\Omega)$. Since $\tilde{\sigma}$ is invariant on the measurable space $(\Omega, \widetilde{\mathcal{B}}(K))$, the positive linear functional,

$$
\Lambda_{T}(\psi)=\frac{1}{2 T} \int_{-T}^{T}\left\{\int_{\Omega} \psi(\omega+t) d \lambda(\omega)\right\} d t, \quad T>0
$$

is also an extension of $\Upsilon$. Observe that $\left\|\Lambda_{T}\right\|=\Lambda_{T}(1)=1$. Then the BanachAlaoglu theorem assures the existence of an accumulation point $\Lambda$ of $\left\{\Lambda_{T} ; T>0\right\}$ in the weak-* topology. We see easily that $\Lambda$ is represented by an invariant probability measure $\nu$ in $M(\Omega)$.

To find a desired ergodic measure, let $K(\Omega)$ be the nonempty set of all invariant probability measures in $M(\Omega)$. Then $K(\Omega)$ is a compact convex subset of $M(\Omega)$ in the weak-* topology. We easily see that a measure in $K(\Omega)$ is ergodic if and only if it is an extreme point of $K(\Omega)$ (refer to the proof of [4, Theorem 6]). Since the above $\nu$ satisfies (2.3) for $\theta$, the Krein-Milman theorem assures the existence of an extreme point $\mu$ of $K(\Omega)$ such that (2.2) holds.

It happens occasionally that the zero-set of $\theta \circ \pi$ contains a nonempty closed invariant set, on which an invariant probability measure is concentrated. This indicates that the conclusion cannot hold for all of the ergodic measures in $K(\Omega)$.

It follows from Wiener's Tauberian theorem that, for a function $f$ in $L^{\infty}(\mathbf{R})$,

$$
a=\lim _{T \rightarrow \infty} \frac{1}{2 T} \int_{-T}^{T} f(t) d t
$$

if and only if

$$
a=\lim _{r \rightarrow \infty} f * P_{i r}(0),
$$

where $f * P_{\text {ir }}$ is the convolution in the usual sense (see the proof of $[15$, Lemma 2.6]). Combining with the individual ergodic theorem, we thus obtain the following:

Lemma 2.2. Let $\mu$ be an invariant, ergodic, probability measure in $M(\Omega)$. If $\phi$ is a function in $L^{\infty}(\mu)$, then we have the equation

$$
\lim _{r \rightarrow \infty} \phi * P_{i r}(\omega)=\int_{\Omega} \phi d \mu, \quad \mu \text {-a.e. } \omega \in \Omega .
$$

From now on, we always assume that each flow we deal with has the following special form. A discrete flow is a pair $\left(\Omega_{0}, U\right)$ consisting of a compact Hausdorff space $\Omega_{0}$ and a homeomorphism $U$ of $\Omega_{0}$ onto itself. Let $\Omega$ be the quotient space obtained from $\Omega_{0} \times[0,1]$ by identifying each $(y, 1)$ with $(U y, 0)$. Under this condition, the flow $\left(\Omega,\left\{U_{t}\right\}_{t \in \mathbf{R}}\right)$ is defined by

$$
U_{t}(y, s)=\left(U^{[t+s]} y, t+s-[t+s]\right), \quad(y, s) \in \Omega,
$$

where $[t]$ denotes the largest integer not exceeding $t$. The flow $\left(\Omega,\left\{U_{t}\right\}_{t \in \mathbf{R}}\right)$ is succinctly said to be built from $\left(\Omega_{0}, U\right)$. We also assume that $\left(\Omega_{0}, U\right)$ has no periodic points; that is, the $\mathbf{Z}$-action determined by $U$ is free. In this event, $\left(\Omega,\left\{U_{t}\right\}_{t \in \mathbf{R}}\right)$ is also free.

Recall that an eigenfunction for a flow $\left(\Omega,\left\{U_{t}\right\}_{t \in \mathbf{R}}\right)$ with eigenvalue $\lambda$ is a Borel function $\psi$ on $\Omega$ such that

$$
U_{t} \psi(\omega)=\psi(\omega+t)=e^{i \lambda t} \psi(\omega) .
$$


A given flow has the form above if and only if there is a continuous eigenfunction with eigenvalue $2 \pi$.

Let $\mu$ be an invariant, ergodic, probability measure in $M(\Omega)$, and let $m_{\mathbf{R}}$ be Lebesgue measure on $\mathbf{R}$. We denote by $m_{I}$ the restriction of $m_{\mathbf{R}}$ to $[0,1)$. Then $\mu$ is represented as $\mu=\mu_{0} \times m_{I}$, where $\mu_{0}$ is the invariant probability measure on $\left(\Omega_{0}, U\right)$ defined by

$$
\mu_{0}(A)=\mu(A \times[0,1)), \quad A \in \mathcal{B}\left(\Omega_{0}\right) .
$$

We next define the operator $P$ of $L^{1}\left(\Omega_{0} \times \mathbf{R}, \mu_{0} \times m_{\mathbf{R}}\right)$ onto $L^{1}(\mu)$ by

$$
P(f)(y, s)=\sum_{n=-\infty}^{\infty} f\left(U^{-n} y, s+n\right), \quad(y, s) \in \Omega .
$$

Observe that $P$ is a bounded linear operator of $L^{1}\left(\mu_{0} \times m_{\mathbf{R}}\right)$ onto $L^{1}(\mu)$. Let $\mathbb{H}^{1}$ be the space of all functions $f$ in $L^{1}\left(\mu_{0} \times m_{\mathbf{R}}\right)$ such that $t \rightarrow f(y, t)$ lies in $H^{1}(\mathbf{R})$ for $\mu_{0}$-a.e. $y \in \Omega_{0}$. Then we see that $P\left(\mathbb{H}^{1}\right)$ is a subspace of $H_{0}^{1}(\mu)$. In more general settings, the operator $P$ defined here and its properties are developed in [1]. Each function $\phi$ on $\Omega$ has the automorphic extension $\phi^{\#}$ to $\Omega_{0} \times \mathbf{R}$ defined by

$$
\phi^{\#}(y, t)=\phi\left(U^{[t]} y, t-[t]\right), \quad(y, t) \in \Omega_{0} \times \mathbf{R} .
$$

Let us show that the subspace $P\left(\mathbb{H}^{1}\right)$ above is dense in $H_{0}^{1}(\mu)$. To see this, suppose that $\psi$ in $L^{\infty}(\mu)$ is orthogonal to $P\left(\mathbb{H}^{1}\right)$. If $f$ lies in $H^{1}(\mathbf{R})$, then $(y, t) \rightarrow p(y) f(t)$ lies in $\mathbb{H}^{1}$ for each $p$ in $L^{1}\left(\mu_{0}\right)$. Then the function $t \rightarrow \psi^{\#}(y, t)$ is orthogonal to $f$ for $\mu_{0}$-a.e. $y \in \Omega_{0}$. Since $H^{1}(\mathbf{R})$ is separable, the function $t \rightarrow \psi^{\#}(y, t)$ lies in $H^{\infty}(\mathbf{R})$ for $\mu_{0}$-a.e. $y \in \Omega_{0}$. This implies that $\psi$ lies in $H^{\infty}(\mu)$, consequently $\psi$ is orthogonal to $H_{0}^{1}(\mu)$. However, more is true; with the aid of the closed range theorem, it is not difficult to show that $P$ maps $\mathbb{H}^{1}$ onto $H_{0}^{1}(\mu)$.

The next lemma is a weak version of [6, Chapter VII, Theorem 7.8].

Lemma 2.3. Let $\mu$ be an invariant, ergodic, probability measure in $M(\Omega)$, and let $1 \leq p \leq \infty$. Then the following are equivalent:

(i) $H_{0}^{p}(\mu)$ has a single generator.

(ii) There is a function $\phi$ in $H^{\infty}(\mu)$ such that $\log |\phi|$ does not lie in $L^{1}(\mu)$ and the function $t \rightarrow \phi(\omega+t)$ is an outer function in $H^{\infty}(\mathbf{R})$ for $\mu$-a.e. $\omega \in \Omega$.

Proof. (ii) implies (i). From the hypotheses of (ii), it follows that $\mathbb{H}^{1} \cdot \phi^{\#}$ is dense in $\mathbb{H}^{1}$. Since $P\left(\mathbb{H}^{1}\right) \cdot \phi$ is dense in $P\left(\mathbb{H}^{1}\right)$, so is $H_{0}^{1}(\mu) \cdot \phi$ in $H_{0}^{1}(\mu)$ by the preceding remark. On the other hand, since $\log |\phi|$ is not summable, Szegö's theorem shows that $\phi$ lies in $H_{0}^{1}(\mu)$. Then $A(\Omega) \cdot \phi$ is dense in $H_{0}^{1}(\mu)$. Since $A(\Omega)$ is a weak-* Dirichlet algebra in $L^{\infty}(\mu)$, it is known that $H_{0}^{1}(\mu) \cap L^{p}(\mu)=H_{0}^{p}(\mu), 1 \leq p \leq \infty$, (see [19]). So $\phi$ is a single generator of $H_{0}^{p}(\mu)$.

(i) implies (ii). Suppose that $H_{0}^{p}(\mu)$ is generated by one element $\phi$ of $H_{0}^{p}(\mu)$. By Szegö's theorem, there is an outer function $\psi$ in $H^{\infty}(\mu)$ with $|\psi|=\min \left(1,|\phi|^{-1}\right)$. So we may assume that $\phi$ lies in $H_{0}^{\infty}(\mu)$ by replacing $\phi$ with $\psi \cdot \phi$. Since $H_{0}^{1}(\mu)$ is the closure of $H_{0}^{p}(\mu)$ in $L^{1}(\mu), H_{0}^{1}(\mu)$ is also singly generated by $\phi$.

Let $q_{0}(t)$ be an inner function in $H^{\infty}(\mathbf{R})$. Regarding $q_{0}$ as a function in $H^{\infty}\left(\mathbf{R}_{+}^{2}\right)$, we assume that $0<q_{0}(i)<1$. It is easy to choose an $r$ with $0<r<1$ such that $2-r\left(q_{0}+q_{0}^{-1}\right)(i)=0$. Then the positive function $f(t)=\left\{2-r\left(q_{0}+\bar{q}_{0}\right)(t)\right\} /\left(1+t^{2}\right)$ in $L^{1}(\mathbf{R})$ is orthogonal to $q_{0} \cdot H^{\infty}(\mathbf{R})$. 
Let $\phi^{\#}(y, t)$ be the automorphic extension of $\phi$ to $\Omega_{0} \times \mathbf{R}$. Since $\phi$ lies in $H_{0}^{\infty}(\mu)$, we obtain the inequality

$$
\int_{-\infty}^{\infty} \log \left|\phi^{\#}(y, t)\right| \frac{d t}{1+t^{2}}>-\infty, \quad \mu_{0} \text {-a.e. } y \in \Omega_{0} .
$$

We notice that $\log \left|\phi^{\#}(y, t)\right|$ is $\mu_{0} \times m_{\mathbf{R}}$-measurable on $\Omega \times \mathbf{R}$. It follows from the formula for outer functions that there is a function $h(y, t)$ in $L^{\infty}\left(\mu_{0} \times m_{\mathbf{R}}\right)$ such that $\left|\phi^{\#}(y, t)\right|=|h(y, t)|$ and such that $t \rightarrow h(y, t)$ is outer in $H^{\infty}(\mathbf{R})$, for $\mu_{0}$-a.e. $y \in \Omega_{0}$. Let us define the unitary function $q$ in $L^{\infty}\left(\mu_{0} \times m_{\mathbf{R}}\right)$ by

$$
q(y, t)=\phi^{\#}(y, t) \cdot h^{-1}(y, t), \quad(y, t) \in \Omega_{0} \times \mathbf{R} .
$$

We then extend $q$ to $\Omega_{0} \times \mathbf{R}_{+}^{2}$ by

$$
q(y, z)=q(y, t+i r)=\int_{-\infty}^{\infty} q(y, t-s) P_{i r}(s) d s .
$$

We claim that $t \rightarrow q(y, t)$ is constant for $\mu_{0}$-a.e. $y \in \Omega_{0}$. Assuming the contrary, the ergodicity implies that $|q(y, t+i)|<1$ for $\mu_{0} \times m_{\mathbf{R}}$-a.e. $(y, t) \in \Omega \times \mathbf{R}$. It follows from Fubini's theorem and the uniqueness theorem of analytic functions that if we fix a suitable $t$, then $0<|q(y, t+i)|<1$ on a subset $E_{0}$ of $\Omega_{0}$ with $\mu_{0}\left(E_{0}\right)>0$. Together with the above observation, we easily construct a nonnegative function $g(y, t)$ in $L^{1}\left(\mu_{0} \times m_{\mathbf{R}}\right)$ for which $\phi^{\#} \cdot g(y, t)$ lies in $\mathbb{H}^{1}$. If $\mu_{0}\left(E_{0}\right)$ is sufficiently small, then the nonnegative function $w=P(g)$ is not constant. Since $\phi \cdot w$ is in $H_{0}^{1}(\mu), \phi$ cannot be a single generator of $H_{0}^{1}(\mu)$. This is a contradiction.

It is a consequence of Szegö's theorem that $\log |\phi|$ cannot lie in $L^{1}(\mu)$.

We add a remark on the condition (ii) of Lemma 2.3. Suppose that a function $\phi$ in $H^{\infty}(\mu)$ satisfies that $t \rightarrow \phi(\omega+t)$ is outer in $H^{\infty}(\mathbf{R})$ for $\mu$-a.e. $\omega \in \Omega$. By the definition of outer function and Lemma 2.2, each of the following properties implies the others:

(a) $\log |\phi|$ does not lie in $L^{1}(\mu)$.

(b) $\int_{\Omega} \phi d \mu=0$.

(c) $\lim _{r \rightarrow \infty} \phi * P_{i r}(\omega)=0, \mu$-a.e. $\omega \in \Omega$.

We now construct a flow which plays an important role in what follows. Let $\beta \mathbf{Z}$ be the Stone-Čech compactification of the group $\mathbf{Z}$ of integers. The shift operator $S_{0}$ of $\mathbf{Z}$ is defined by $S_{0} n=n+1$. Then $S_{0}$ induces naturally an isometric isomorphism of $\ell^{\infty}(\mathbf{Z})$ onto itself, where $\ell^{\infty}(\mathbf{Z})$ is the algebra of all bounded sequences on $\mathbf{Z}$. Since $\beta \mathbf{Z}$ is the maximal ideal space of $\ell^{\infty}(\mathbf{Z})$, it follows from the BanachStone theorem [3, Chapter IV, 6.26] that $S_{0}$ extends to a homeomorphism $S$ of $\beta \mathbf{Z}$ onto itself. Then the pair $(\beta \mathbf{Z}, S)$ makes a discrete flow; thus we obtain the flow $\left(X,\left\{S_{t}\right\}_{t \in \mathbf{R}}\right)$ built from $(\beta \mathbf{Z}, S)$.

Let $X_{0}$ be the quotient space obtained from $\mathbf{Z} \times[0,1]$ by identifying each $(n, 1)$ with $(n+1,0)$. Since $\mathbf{Z}$ is dense in $\beta \mathbf{Z}$, so is $X_{0}$ in $X$. We also see that $\mathbf{R}$ is homeomorphic to $X_{0}$ via the map $t \rightarrow([t], t-[t])$. Let $C_{b u}(\mathbf{R})$ be the space of all bounded uniformly continuous functions on $\mathbf{R}$. Since $X$ is compact, each function in $C(X)$ determines uniquely a function in $C_{b u}(\mathbf{R})$, by restricting it to $X_{0}$.

The converse also holds (compare with [18, Lemma 2.1]).

Lemma 2.4. Let $f$ be a function in $C_{b u}(\mathbf{R})$. Then $f$ extends uniquely to a function $\Psi(f)$ in $C(X)$, and the map $\Psi$ is an equivariant, isometric, isomorphism of $C_{b u}(\mathbf{R})$ 
onto $C(X)$ satisfying the equation

$$
\Psi\left(C_{b u}(\mathbf{R}) \cap H^{\infty}(\mathbf{R})\right)=A(X) .
$$

Proof. We may regard $f$ as a function on $X_{0}$. If we fix an $s$ in $[0,1)$, then the sequence $\{f(n, s)\}$ belongs to $\ell^{\infty}(\mathbf{Z})$. So the function $n \rightarrow f(n, s)$ extends to a function $y \rightarrow \Psi(f)(y, s)$ in $C(\beta \mathbf{Z})$. Since $X$ is identified with $\beta \mathbf{Z} \times[0,1)$, we obtain a function $\Psi(f)(y, s)$ on $X$. We have to show that $\Psi(f)(y, s)$ is continuous on $X$. Let $\Psi^{\#}(f)(y, t)$ be the automorphic extension of $\Psi(f)(y, s)$ to $\beta \mathbf{Z} \times \mathbf{R}$. Since $f(t)$ is uniformly continuous on $\mathbf{R}$, the family $\left\{t \rightarrow \Psi(f)^{\#}(y, t) ; y \in \beta \mathbf{Z}\right\}$ is equicontinuous on $\mathbf{R}$. This implies that $\Psi(f)^{\#}(y, t)$ is continuous on $\beta \mathbf{Z} \times \mathbf{R}$; so $\Psi(f)$ lies in $C(X)$.

Since $\Psi(f)$ equals $f$ on the dense subset $X_{0}, \Psi(f)$ is uniquely determined and satisfies the equality $\|\Psi(f)\|_{\infty}=\|f\|_{\infty}$. In view of the preceding remark, we see that $\Psi$ is an equivariant, isometric, isomorphism of $C_{b u}(\mathbf{R})$ onto $C(X)$. Observe that $\Psi(f * g)=\Psi(f) * g$ for each $g$ in $L^{1}(\mathbf{R})$. From this, (2.4) follows easily.

The interest in the flow $\left(X,\left\{S_{t}\right\}_{t \in \mathbf{R}}\right)$ lies in the fact that each outer function in $C_{b u}(\mathbf{R}) \cap H^{\infty}(\mathbf{R})$ extends to a function in $A(X)$ with similar properties.

\section{Representation of ALMOST PERIODiC FunCtions}

Let $\Gamma, e_{t},\left(K,\left\{T_{t}\right\}_{t \in \mathbf{R}}\right)$, and $\sigma$ be as in Section 1. There is no loss of generality in assuming that $\Gamma$ contains $2 \pi$, and $K_{2 \pi}$ denotes the closed subgroup of $K$ of all $x$ such that $\chi_{2 \pi}(x)=1$. We define the homeomorphism $T$ of $K_{2 \pi}$ onto itself by $T(y)=y+e_{1}$. Then $\left(K,\left\{T_{t}\right\}_{t \in \mathbf{R}}\right)$ is represented as the flow built from the discrete flow $\left(K_{2 \pi}, T\right)$. Let $\sigma_{0}$ be the normalized Haar measure on $K_{2 \pi}$. Then $\sigma$ is represented as $\sigma=\sigma_{0} \times m_{I}$.

Let $B_{\Gamma}(\mathbf{R})$ denote the space of all uniformly almost periodic functions with exponents in $\Gamma$. Then $B_{\Gamma}(\mathbf{R})$ is a selfadjoint closed subalgebra of $C_{b u}(\mathbf{R})$. We fix a point $p$ in $K$. If $\phi$ is in $C(K)$, then $t \rightarrow \phi(p+t)$ lies in $B_{\Gamma}(\mathbf{R})$. Conversely, each function in $B_{\Gamma}(\mathbf{R})$ is obtained in this way. From Lemma 2.4, it follows that $\Psi\left(B_{\Gamma}(\mathbf{R})\right)$ is also a selfadjoint closed subalgebra of $C(X)$, which does not separate the points of $X$. Since $B_{\Gamma}(\mathbf{R})$ is translation-invariant and $\Psi$ is equivariant, it follows that $\Psi\left(B_{\Gamma}(\mathbf{R})\right)$ is invariant under the group $\left\{S_{t}\right\}_{t \in \mathbf{R}}$.

Define the isometric isomorphism $\Pi_{p}$ of $C(K)$ onto $\Psi\left(B_{\Gamma}(\mathbf{R})\right)$ by

$$
\Pi_{p} \phi=\Psi(\phi(p+t)), \quad \phi \in C(K) .
$$

We then see the equation

$$
\Pi_{p}\left(T_{t} \phi\right)=S_{t}\left(\Pi_{p} \phi\right), \quad t \in \mathbf{R} .
$$

By the Banach-Stone theorem, there is a continuous map $\pi_{p}$ of $X$ onto $K$ such that $\Pi_{p} \phi=\phi \circ \pi_{p}$ and

$$
T_{t} \circ \pi_{p}(\omega)=\pi_{p} \circ S_{t}(\omega), \quad \omega \in X .
$$

Thus $\left(X,\left\{S_{t}\right\}_{t \in \mathbf{R}}\right)$ is an extension of $\left(K,\left\{T_{t}\right\}_{t \in \mathbf{R}}\right)$ under the map $\pi_{p}$. By (2.1), the map $\pi_{p}$ induces $\pi_{p}^{-1}(\mathcal{B}(K)), \widetilde{C}(K)$, and $\widetilde{A}(K)$. We then observe that

$$
\left\{\begin{array}{l}
\widetilde{C}(K)=\Pi_{p}(C(K))=\Psi\left(B_{\Gamma}(\mathbf{R})\right), \\
\widetilde{A}(K)=\Pi_{p}(A(K))=\Psi\left(B_{\Gamma}(\mathbf{R}) \cap H^{\infty}(\mathbf{R})\right) .
\end{array}\right.
$$

Let $\tilde{\sigma}$ be the probability measure on the $\sigma$-algebra $\pi_{p}^{-1}(\mathcal{B}(K))$ defined by

$$
\tilde{\sigma}(E)=\sigma\left(\pi_{p}(E)\right), \quad E \in \pi_{p}^{-1}(\mathcal{B}(K)),
$$


as before. Then $L^{\infty}(\sigma)$ is identified with $L^{\infty}\left(X, \pi_{p}^{-1}(\mathcal{B}(K)), \tilde{\sigma}\right)$ via the map $\phi \rightarrow$ $\phi \circ \pi_{p}$.

If $\phi$ is a function in $C(K)$, then

$$
\tilde{\sigma}\left(\left\{\omega ; \Pi_{p} \phi(\omega) \leq s\right\}\right)=\sigma(\{x ; \phi(x) \leq s\}), \quad s \in \mathbf{R} .
$$

In order to extend the relation to suitable functions in $L^{\infty}(\sigma)$, we introduce a certain $\sigma$-subalgebra $\widetilde{\mathcal{B}}(K)$ of $\mathcal{B}(X)$ containing $\pi_{p}^{-1}(\mathcal{B}(K))$. Let $J$ be a subset of $\mathbf{Z}$. The density $\alpha(J)$ of $J$ is defined by

$$
\alpha(J)=\limsup _{n \rightarrow \infty} \frac{1}{2 n+1} \sum_{j=-n}^{n} \chi_{J}(j),
$$

where $\chi_{J}$ denotes the characteristic function of $J$. Since the closure $\operatorname{cl}[J]$ of $J$ in $\beta \mathbf{Z}$ is an open and closed set in $\beta \mathbf{Z}$ (see $[20]), \operatorname{cl}[J] \times[0,1)$ is an element of $\mathcal{B}(X)$. Let $\left\{J_{k}\right\}$ be a decreasing sequence of subsets of $\mathbf{Z}$ such that $\alpha\left(J_{k}\right) \rightarrow 0$. Define

$$
\left\{\begin{aligned}
A_{k} & =\left(\operatorname{cl}\left[J_{k}\right] \backslash J_{k}\right) \times[0,1), \\
A & =\bigcap_{k=1}^{\infty} A_{k} .
\end{aligned}\right.
$$

Since $J_{k}$ is open in $\beta \mathbf{Z}, A$ lies also in $\mathcal{B}(X)$. If each $J_{k}$ is infinite, then $A$ is not empty by the finite intersection property. We denote by $\mathcal{D}$ the collection of all such sets $A$ in $\mathcal{B}(X)$. If $\left\{A^{(n)}\right\}$ is a sequence in $\mathcal{D}$, then $\bigcup_{n=0}^{\infty} A^{(n)}$ is contained in some element of $\mathcal{D}$.

Let $\widetilde{\mathcal{D}}$ denote the collection of all $B$ in $\mathcal{B}(X)$ where $B$ is a subset of an element of $\mathcal{D}$. Then $\widetilde{\mathcal{D}}$ is closed under countable unions and translations by $\left\{S_{t}\right\}_{t \in \mathbf{R}}$. We denote by $\widetilde{\mathcal{B}}(K)$ the $\sigma$-subalgebra generated by $\pi_{p}^{-1}(\mathcal{B}(K))$ and $\widetilde{\mathcal{D}}$. Then $E$ lies in $\widetilde{\mathcal{B}}(K)$ if and only if there is an $E_{1}$ in $\pi_{p}^{-1}(\mathcal{B}(K))$ such that $E \Delta E_{1}$ lies in $\widetilde{\mathcal{D}}$, where $E \Delta E_{1}$ is the symmetric difference of $E$ and $E_{1}$. We may extend $\tilde{\sigma}$ to $\widetilde{\mathcal{B}}(K)$ by $\tilde{\sigma}(E)=\tilde{\sigma}\left(E_{1}\right)$. Indeed, let $F$ be a compact set in $K$ with $\sigma(F)>0$, and let $A$ be an element of $\mathcal{D}$. Then the Borel set $\pi_{p}^{-1}(F) \backslash A$ is never empty, and this makes sense to extend $\tilde{\sigma}$ to $\widetilde{\mathcal{B}}(K)$. Suppose that $\phi$ is a function in $C(K)$ such that $0 \leq \phi \leq 1$ on $K$, while $\phi=1$ on $F$. Since $K$ is completely regular, $F$ is an intersection of $\phi^{-1}(\{1\})$ of such functions $\phi$. Recall that $\left(K,\left\{T_{t}\right\}_{t \in \mathbf{R}}\right)$ is strictly ergodic. Then, for a fixed point $p$ in $K$, we see that

$$
\lim _{T \rightarrow \infty} \frac{1}{2 T} \int_{-T}^{T} \phi(p+t) d t>\sigma(F)>0 .
$$

Let $\left\{J_{k}\right\}$ and $\left\{A_{k}\right\}$ be as in (3.4). Since $\alpha\left(J_{k}\right) \rightarrow 0$, if we choose a large $k$, then $\left\{\omega ; \Pi_{p} \phi(\omega)=1\right\} \backslash A_{k}$ is not empty for all $\phi$. From this and the compactness of $X$, it follows easily that $\pi_{p}^{-1}(F) \backslash A$ is not empty. Since the measure algebras $\langle\widetilde{\mathcal{B}}(K), \tilde{\sigma}\rangle$ and $\left\langle\pi_{p}^{-1}(\mathcal{B}(K)), \tilde{\sigma}\right\rangle$ are isomorphic in the sense of Boolean algebras, $L^{\infty}(X, \widetilde{\mathcal{B}}(K), \tilde{\sigma})$ is isometrically isomorphic to $L^{\infty}(\sigma)$.

We next consider a suitable subspace of $L^{\infty}(\sigma)$ to which (3.1) may be extended. Let $L_{b u}^{\infty}(\sigma)$ be the space of all functions $\psi$ in $L^{\infty}(\sigma)$ such that $t \rightarrow \psi(x+t)$ lies in $C_{b u}(\mathbf{R})$ for $\sigma$-a.e. $x \in K$. Then the equation $\Pi_{p} \psi=\Psi(\psi(p+t))$ in (3.1) is defined for each $p$ outside a $\sigma$-null set in $K$. Observe that if $\phi$ is in $L^{\infty}(\sigma)$, then $\phi * P_{i r}$, $r>0$, lies in $L_{b u}^{\infty}(\sigma)$. 
Let $\varepsilon>0$. For a function $\psi$ in $L_{b u}^{\infty}(\sigma)$, there is a $\delta>0$ for which the invariant set,

$$
H(\varepsilon)=\left\{x \in K ; \sup _{|t-s|<\delta}|\psi(x+t)-\psi(x+s)| \leq \varepsilon\right\},
$$

has positive $\sigma$-measure. Since $\sigma$ is ergodic, we obtain $\sigma(H(\varepsilon))=1$. By choosing an invariant $\sigma$-null set $N_{0}$, containing $\bigcup_{n=1}^{\infty} H\left(\frac{1}{n}\right)^{c}$, the family $\{t \rightarrow \psi(x+t) ; x \in$ $\left.K \backslash N_{0}\right\}$ in $C_{b u}(\mathbf{R})$ is equicontinuous and uniformly bounded on $\mathbf{R}$. This implies that

$$
\lim _{r \rightarrow 0}\left\{\sup _{t \in \mathbf{R}}\left|\psi(x+t)-\psi * P_{i r}(x+t)\right|\right\}=0
$$

holds uniformly on the invariant set $K \backslash N_{0}$.

Lemma 3.1. Let $\psi$ be a function in $L_{b u}^{\infty}(\sigma)$. Then there is an invariant $\sigma$-null set $N$, depending on $\psi$, such that, if we fix a point $p$ in $K \backslash N$, then $\Pi_{p} \psi$ belongs to $C(X) \cap L^{\infty}(X, \widetilde{\mathcal{B}}(K), \tilde{\sigma})$ and satisfies the equations

$$
\Pi_{p} \psi(\omega)=\psi \circ \pi_{p}(\omega), \quad \tilde{\sigma} \text {-a.e. } \omega \in X,
$$

and

$$
\int_{X} \Pi_{p} \psi d \tilde{\sigma}=\int_{K} \psi d \sigma
$$

Proof. We first claim that there is a sequence $\left\{\phi_{j}\right\}$ in $C(K)$ with $\left\|\phi_{j}\right\|_{\infty} \leq\|\psi\|_{\infty}$ such that

$$
\lim _{j \rightarrow \infty}\left\{\sup _{0 \leq t \leq 1}\left|\phi_{j}(x+t)-\psi(x+t)\right|\right\}=0, \quad x \in K \backslash N_{1},
$$

where $N_{1}$ is an invariant $\sigma$-null set. Indeed, suppose that $\left\{\psi_{k}\right\}$ is a sequence in $C(K)$ with $\left\|\psi_{k}\right\|_{\infty} \leq\|\psi\|_{\infty}$ such that

$$
\lim _{k \rightarrow \infty} \psi_{k}(x)=\psi(x), \quad \sigma \text {-a.e. } x \in K \text {. }
$$

By Fubini's theorem, we see that

$$
\lim _{k \rightarrow \infty} \psi_{k}(x+s)=\psi(x+s), \quad m_{\mathbf{R}} \text {-a.e. } s \in \mathbf{R},
$$

for $\sigma$-a.e. $x \in K$. Since

$$
\sup _{0 \leq t \leq 1} P_{i r}(s-t) \leq C P_{i r}(s)
$$

for some constant $C>0$, this implies

$$
\lim _{k \rightarrow \infty}\left\{\sup _{0 \leq t \leq 1}\left|\psi_{k} * P_{i r}(x+t)-\psi * P_{i r}(x+t)\right|\right\}=0, \quad r>0 .
$$

Observe that this also converges in probability. Let $\{r(j)\}$ be a decreasing sequence such that $r(j) \rightarrow 0$. By setting $\phi_{j}=\psi_{j} * P_{i r(j)}$ for some subsequence $\left\{\psi_{j}\right\}$ of $\left\{\psi_{k}\right\}$, (3.8) follows from (3.5). 
For a given $\varepsilon>0$, we set

$$
\begin{aligned}
E(n) & =\left\{x \in K ; \sup _{j, k \geq n}\left|\phi_{j}(x)-\phi_{k}(x)\right| \leq \varepsilon\right\} \\
& =\bigcap_{j, k \geq n}\left\{x \in K ;\left|\phi_{j}(x)-\phi_{k}(x)\right| \leq \varepsilon\right\} .
\end{aligned}
$$

Then the sequence $\{E(n)\}$ of closed sets is increasing and satisfies

$$
\lim _{n \rightarrow \infty} \sigma(E(n))=1
$$

since $\left\{\phi_{j}(x)\right\}$ converges for $\sigma$-a.e. $x \in K$. For a fixed point $p$ in $K$, we then define

$$
E_{1}(n)=\bigcap_{j, k \geq n}\left\{w \in X ;\left|\Pi_{p} \phi_{j}(\omega)-\Pi_{p} \phi_{k}(\omega)\right| \leq \varepsilon\right\} .
$$

Since $\left|\phi_{j}-\phi_{k}\right|$ lies in $C(K)$, we observe $E_{1}(n)=\pi_{p}^{-1}(E(n))$. This shows that $E_{1}(n)$ lies in $\widetilde{\mathcal{B}}(K)$ and $\tilde{\sigma}\left(E_{1}(n)\right)=\sigma(E(n))$. Since $\lim _{n \rightarrow \infty} \sigma\left(E(n) \backslash N_{1}\right)=1$, it follows from (3.8) that there is a subsequence $\left\{n_{k}\right\}$ of $\{n\}$ and an increasing sequence $\{W(k)\}$ of compact sets in $K_{2 \pi}$ such that $E\left(n_{k}\right) \backslash N_{1}$ contains $W(k) \times[0,1)$ and $\sigma(W(k) \times[0,1))>1-2^{-k}$. By virtue of the individual ergodic theorem, we find an invariant $\sigma$-null set $N_{2}$ such that, if the point $p$ lies in $K \backslash N_{2}$, then

$$
\lim _{T \rightarrow \infty} \frac{1}{2 T} \int_{-T}^{T} \chi_{W(k) \times[0,1)}(p+t) d t>1-2^{-k},
$$

for $k=1,2, \ldots$. Recall that $p$ is written as $(q, s)$ in $K_{2 \pi} \times[0,1)$. We then define that

$$
J_{k}=\{n \in \mathbf{Z} ; q+n \notin W(k)\},
$$

and let $\left\{A_{k}\right\}$ and $A$ be as in (3.4). Since the density $\alpha\left(J_{k}\right)$ satisfies the inequality $\alpha\left(J_{k}\right)<2^{-k}$ by (3.9), the set $A$ is a $\tilde{\sigma}$-null set in $\widetilde{\mathcal{B}}(K)$ by the definition of $\tilde{\sigma}$. If we choose a point $p$ in $K \backslash\left(N_{1} \cup N_{2}\right)$, then

$$
\sup _{j \geq n_{k}}\left|\Pi_{p} \psi(\omega)-\Pi_{p} \phi_{j}(\omega)\right| \leq \varepsilon, \quad \omega \in E_{1}\left(n_{k}\right) \backslash A_{k} .
$$

Indeed, when $p+t$ is in $W(k) \times[0,1)$, we see by (3.8) the inequality

$$
\sup _{j \geq n_{k}}\left|\psi(p+t)-\phi_{j}(p+t)\right| \leq \varepsilon \text {. }
$$

Since $\left\{E\left(n_{k}\right)\right\}$ is increasing and $\left\{A_{k}\right\}$ is decreasing, we obtain

$$
\bigcup_{k=1}^{\infty}\left(E_{1}\left(n_{k}\right) \backslash A_{k}\right)=\left(\bigcup_{k=1}^{\infty} E_{1}\left(n_{k}\right)\right) \backslash\left(\bigcap_{k=1}^{\infty} A_{k}\right) \text {. }
$$

So the left-side set lies in $\widetilde{\mathcal{B}}(K)$ and its $\tilde{\sigma}$-measure is 1 . Since $\varepsilon>0$ is arbitrary, there is an invariant $\sigma$-null set $N$, containing $N_{1} \cup N_{2}$, such that, for a fixed $p$ in $K \backslash N$,

$$
\lim _{j \rightarrow \infty} \Pi_{p} \phi_{j}(\omega)=\Pi_{p} \psi(\omega), \quad \tilde{\sigma} \text {-a.e. } \omega \in X .
$$

Therefore, $\Pi_{p} \psi$ belongs to $C(X) \cap L^{\infty}(X, \widetilde{\mathcal{B}}(K), \tilde{\sigma})$.

On the other hand, since $\Pi_{p} \phi_{j}(\omega)=\phi_{j} \circ \pi_{p}(\omega)$ on $X$ and

$$
\lim _{j \rightarrow \infty} \phi_{j}(x)=\psi(x), \quad \sigma \text {-a.e. } x \in K,
$$


(3.6) follows from (3.10), and (3.7) is an immediate consequence of (3.6).

\section{Extension of $\left(K,\left\{T_{t}\right\}_{t \in \mathbf{R}}\right)$}

It is known that $H^{\infty}(\sigma)$ contains a nonnull function $\phi$ for which $\log |\phi|$ is not summable. Recall that the equation in (1.1) is represented as $\rho(\phi)(x)=(\log |\phi|) *$ $P_{i}(x)$. Since $\phi$ is analytic, it satisfies automatically that $\rho(\phi)(x)>-\infty$ on $K$ (see $[9$, Chapter $3, \S 3$ and $\S 4])$. We deal with the moduli of such analytic functions.

Let $\mathbf{W}^{\infty}$ be the family of all nonnegative functions $w$ in $L^{\infty}(\sigma)$ such that $\log w$ does not lie in $L^{1}(\sigma)$, while $\rho(w)(x)>-\infty$ for $\sigma$-a.e. $x \in K$. For a function $w$ in $\mathbf{W}^{\infty}$, and for an $r>0$, we define

$$
w_{r}(x)=\exp \left\{(\log w) * P_{i r}(x)\right\} .
$$

It then follows from Fubini's theorem that $w_{r}$ lies in $\mathbf{W}^{\infty}$. Observe the inequality $0<w_{r}(x)<\|w\|_{\infty}$ on $K$. We now show $w_{r}$ satisfies the hypothesis of Lemma 3.1, that is, $w_{r}$ lies in $L_{b u}^{\infty}(\sigma)$. Indeed, if we take an $x$ outside a $\sigma$-null set, then there is an outer function $h_{x}$ in $H^{\infty}(\mathbf{R})$ such that $\left|h_{x}(t)\right|=w(x+t)$ on $\mathbf{R}$. Since $h_{x}$ is bounded on $\mathbf{R}$, the function $t \rightarrow h_{x} * P_{i r}(t)$ lies in $C_{b u}(\mathbf{R}) \cap H^{\infty}(\mathbf{R})$. From the property of outer functions in $H^{\infty}(\mathbf{R})$, we see that

$$
\left|h_{x} * P_{i r}(t)\right|=\exp \left\{(\log w) * P_{i r}(x+t)\right\}=w_{r}(x+t) .
$$

So $w_{r}$ is an element of $L_{b u}^{\infty}(\sigma)$. Of course, we may show this fact directly.

It follows from Lemma 2.4 that $\Psi\left(h_{x} * P_{i r}\right)$ lies in $A(X)$. Moreover, we see that

$$
\begin{aligned}
\left|\Psi\left(h_{x} * P_{i r}\right)\right| & =\Psi\left(\left|h_{x} * P_{i r}\right|\right), \\
\Psi\left(h_{x} * P_{i r}\right) * P_{i s} & =\Psi\left(h_{x} * P_{i(r+s)}\right), \quad s>0,
\end{aligned}
$$

since $\Psi$ is isometric and $P_{i(r+s)}=P_{i r} * P_{i s}$. Since $w_{(r+1)}$ as well as $w_{r}$ lies in $L_{b u}^{\infty}(\sigma)$ Lemma 3.1 implies that, if we fix a point $p$ outside an invariant $\sigma$-null set $N$, then

$$
\left\{\begin{aligned}
\left|\Psi\left(h_{p} * P_{i r}\right)(\omega)\right| & =w_{r} \circ \pi_{p}(\omega), \\
\left|\Psi\left(h_{p} * P_{i(r+1)}\right)(\omega)\right| & =w_{(r+1)} \circ \pi_{p}(\omega),
\end{aligned}\right.
$$

for $\tilde{\sigma}$-a.e. $\omega \in X$. We note that $\Psi\left(h_{p} * P_{i r}\right)$ itself may not belong to $L^{\infty}(X, \widetilde{\mathcal{B}}(K), \tilde{\sigma})$, although $\left|\Psi\left(h_{p} * P_{i r}\right)\right|$ lies in $L^{\infty}(X, \widetilde{\mathcal{B}}(K), \tilde{\sigma})$ by Lemma 3.1. Since $w_{r}$ lies in $\mathbf{W}^{\infty}$, we see that $\left|\Psi\left(h_{p} * P_{i r}\right)\right|>0$ for $\tilde{\sigma}$-a.e. $\omega \in X$. However, the zero-set of $\left|\Psi\left(h_{p} * P_{i r}\right)\right|$ always contains a nonempty closed invariant set in $\mathcal{B}(X)$. This follows from the fact that $\rho\left(\left|\Psi\left(h_{p} * P_{i r}\right)\right|\right)\left(\omega_{n}\right)$ may tend to $-\infty$ for some sequence $\left\{\omega_{n}\right\}$ in $X$.

Fix an $r>0$, and define the function $\psi_{p}$ in $A(X)$ by

$$
\psi_{p}(\omega)=\Psi\left(h_{p} * P_{i r}\right)(\omega), \quad \omega \in X .
$$

By (4.1) and (4.2), we see that

$$
\begin{aligned}
\left|\psi_{p} * P_{i}(\omega)\right| & =\left|\Psi\left(h_{p} * P_{i(r+1)}\right)(\omega)\right| \\
& =w_{(r+1)} \circ \pi_{p}(\omega) \\
& =\exp \left\{\left(\log w_{r} \circ \pi_{p}\right) * P_{i}(\omega)\right\} \\
& =\exp \left\{\left(\log \left|\psi_{p}\right|\right) * P_{i}(\omega)\right\}
\end{aligned}
$$

for $\tilde{\sigma}$-a.e. $\omega \in X$. Since $\log \left|\psi_{p} * P_{i}(\omega)\right|=\log \left|\psi_{p}\right| * P_{i}(\omega)$, this shows that $t \rightarrow$ $\psi_{p}(\omega+t)$ is an outer function in $H^{\infty}(\mathbf{R})$ outside an invariant $\tilde{\sigma}$-null set. 
Lemma 4.1. Let $\psi_{p}$ be the function in $A(X)$ defined by (4.3). Then there is an invariant $\tilde{\sigma}$-null set $\widetilde{N}$ in $\widetilde{\mathcal{B}}(K)$ such that, for each $\omega$ in $X \backslash \widetilde{N}$, the function $t \rightarrow$ $\psi_{p}(\omega+t)$ is an outer function in $H^{\infty}(\mathbf{R})$ and satisfies the equation

$$
\lim _{s \rightarrow \infty} \psi_{p} * P_{i s}(\omega)=0
$$

Proof. It suffices to show that (4.4) holds for $\tilde{\sigma}$-a.e. $\omega \in X$. For $n=1,2, \ldots$, let $w^{(n)}=\max (w, 1 / n)$. Since $\log w^{(n)}$ is in $L^{1}(\sigma)$, Szegö's theorem assures the existence of an outer function $\phi^{(n)}$ in $H^{\infty}(\sigma)$ such that $\left|\phi^{(n)}\right|=w^{(n)}$. Since $\log w$ does not lie in $L^{1}(\sigma)$, the Fourier coefficient,

$$
a_{0}\left(\phi^{(n)}\right)=\int_{K} \phi^{(n)} d \sigma=\int_{K} \phi^{(n)} * P_{i r} d \sigma
$$

tends to 0 as $n \rightarrow \infty$. Indeed, by the property of outer functions, we see that

$$
\log \mid a_{0}\left(\phi^{(n)} \mid=\int_{K} \log w^{(n)} d \sigma\right.
$$

diverges to $-\infty$. Furthermore, Lemma 2.2 shows that

$$
\lim _{s \rightarrow \infty}\left(\phi^{(n)} * P_{i r}\right) * P_{i s}(x)=a_{0}\left(\phi^{(n)}\right), \quad \sigma \text {-a.e. } x \in K .
$$

Let $N$ be the invariant $\sigma$-null set for which (4.2) holds. By modifying $N$, if necessary, Lemma 3.1 shows that, if we take a point $p$ in $K \backslash N$, then (4.3) is defined and

$$
\lim _{s \rightarrow \infty} \Pi_{p}\left(\phi^{(n)} * P_{i r}\right) * P_{i s}(\omega)=a_{0}\left(\phi^{(n)}\right), \quad \tilde{\sigma} \text {-a.e. } \omega \in X,
$$

for $n=1,2, \ldots$.

On the other hand, since $w(x) \leq w^{(n)}(x)$ and

$$
\left|\left(\phi^{(n)} * P_{i r}\right) * P_{i s}(x)\right|=\exp \left\{\left(\log w^{(n)}\right) * P_{i r} * P_{i s}(x)\right\},
$$

we see that

$$
\left|\psi_{p} * P_{i s}(\omega)\right| \leq\left|\Pi_{p}\left(\phi^{(n)} * P_{i r}\right) * P_{i s}(\omega)\right| .
$$

By (4.5), this yields the inequality

$$
\limsup _{s \rightarrow \infty}\left|\psi_{p} * P_{i s}(\omega)\right| \leq\left|a_{0}\left(\phi^{(n)}\right)\right|, \quad \tilde{\sigma} \text {-a.e. } \omega \in X,
$$

from which (4.4) follows.

Let us introduce an auxiliary function $\theta$ in $L_{b u}^{\infty}(\sigma)$ such that the above $\psi_{p}$ is well-behaved on the support of $\Pi_{p} \theta$. Recall that $K$ and $\sigma$ may be regarded as $K_{2 \pi} \times[0,1)$ and $\sigma_{0} \times m_{I}$, respectively.

By Egoroff's theorem, there is a compact subset $F$ of $K$ with $\sigma(F)>0$ on which (4.4) converges uniformly as $s \rightarrow \infty$. Observe that

$$
\int_{-\infty}^{\infty}\left|P_{i s}(t-u)-P_{i s}(t+u)\right| d t=\frac{2}{\pi s} \arctan \left(\frac{u}{s}\right) .
$$

This ensures that $F$ may have the form $F_{0} \times[1 / 3,2 / 3]$ for a compact subset $F_{0}$ of $K_{2 \pi}$ with $\sigma_{0}\left(F_{0}\right)>0$.

Let $w_{r}$ be the function defined by (4.1). We may also assume that $w_{r}$ is continuous on $F=F_{0} \times[1 / 3,2 / 3]$ and

$$
\rho\left(w_{r}\right)(x)>m, \quad x \in F,
$$


for a constant $m>-\infty$. Indeed, let $\left\{s_{n}\right\}$ be a countable dense set in $[1 / 3,2 / 3]$. Applying Lusin's theorem, we make $y \rightarrow w_{r}\left(y, s_{n}\right)$ continuous on $F_{0}$, for $n=1,2, \ldots$ Since $w_{r}$ lies in $L_{b u}^{\infty}(\sigma)$, we regard $\left\{s \rightarrow w_{r}(y, s) ; y \in F_{0}\right\}$ as an equicontinuous family on $[1 / 3,2 / 3]$, by the remark preceding Lemma 3.1. Then $w_{r}$ satisfies the desired properties on $F=F_{0} \times[1 / 3,2 / 3]$. Moreover, we assume that $w_{(r+1)}$ is also continuous on $F$ and satisfies (4.6) with $r$ replaced by $(r+1)$.

Let $u$ be a continuous function on $[0,1)$ such that $u(s)>0$ on $(1 / 3,2 / 3)$ and $u(s)=0$ otherwise. Define the function $\theta$ in $L^{\infty}(\sigma)$ by

$$
\theta(y, s)=\chi_{F_{0}}(y) \cdot u(s), \quad(y, s) \in K_{2 \pi} \times[0,1) .
$$

Then $\theta$ lies in $L_{b u}^{\infty}(\sigma)$. There is an invariant $\sigma$-null set $N_{0}$ such that the conclusion of Lemma 3.1 holds simultaneously for $\theta, w_{r}$, and $\phi^{(n)} * P_{i r}$ in (4.5), whenever a point $p$ in $K \backslash N_{0}$ is fixed. Then $\Pi_{p} \theta$ is a function in $C(X)$ with compact support $\widetilde{F}=\widetilde{F}_{0} \times[1 / 3,2 / 3]$, where $\widetilde{F}_{0}$ is an open and closed set in $\beta \mathbf{Z}$ (see [20]). We see easily that $\tilde{\sigma}(\widetilde{F})=\sigma(F)>0$ and $\pi_{p}(\widetilde{F})$ is contained in $F$. From this, it follows that the function $\psi_{p}$ given by (4.3) satisfies the conclusion of Lemma 4.1 on $\widetilde{F}$ without exception, that is, for each $\omega$ in $\widetilde{F}, t \rightarrow \psi_{p}(\omega+t)$ is an outer function in $H^{\infty}(\mathbf{R})$ and $\lim _{s \rightarrow \infty} \psi_{p} * P_{i s}(\omega)=0$.

We now offer a proof of Theorem 1.1 stated in Section 1.

Proof of Theorem 1.1. Let $\theta$ and $N_{0}$ be as in the above. Fix a $p$ in $K \backslash N_{0}$, and let $\psi_{p}$ be the function in $A(X)$ given by (4.3). By Lemma 3.1, the function $\Pi_{p} \theta$ in $C(X)$ satisfies

$$
\int_{X} \Pi_{p} \theta d \tilde{\sigma}=\int_{K} \theta d \sigma>0 .
$$

From (3.6), Lemma 2.1 ensures the existence of an invariant, ergodic, probability measure $\mu$ in $M(X)$ such that

$$
\int_{X} \Pi_{p} \theta d \mu>0
$$

Recall that $\Pi_{p} \theta$ is supported on the compact set $\widetilde{F}=\widetilde{F}_{0} \times[1 / 3,2 / 3]$ in $X$. Then (4.8) implies that $\mu(\widetilde{F})>0$. Define the invariant set $\widetilde{E}$ in $X$ by

$$
\widetilde{E}=\bigcup\{\widetilde{F}+t ; t \in \mathbf{R}\}=\bigcup_{t \in \mathbf{R}} S_{t}(\widetilde{F}),
$$

which is open in $X$. Since $\mu$ is ergodic, we see that $\mu(\widetilde{E})=1$. Together with the preceding remark, we obtain that, for $\mu$-a.e. $\omega \in X, t \rightarrow \psi_{p}(\omega+t)$ is an outer function in $H^{\infty}(\mathbf{R})$ and

$$
\lim _{s \rightarrow \infty} \psi_{p} * P_{i s}(\omega)=0 .
$$

Let $H^{p}(X, \mu), 1 \leq p \leq \infty$, denote the Hardy space on $\left(X,\left\{S_{t}\right\}_{t \in \mathbf{R}}\right)$ relative to the above measure $\mu$ (refer to Section 1$)$. Then $H_{0}^{p}(X, \mu)$ has a single generator $\psi_{p}$ by Lemma 2.3 .

The space $H^{p}(X, \mu)$ is very large, much larger often than is necessary. To obtain a smaller example of a space $H_{0}^{p}(\mu)$ that is singly generated, we take a suitable factor of $\left(X,\left\{S_{t}\right\}_{t \in \mathbf{R}}\right)$ constructed as follows. Let $\widetilde{C}(K)$ and $\widetilde{A}(K)$ be as in (3.3). We then denote by $C_{0}$ the selfadjoint closed subalgebra of $C(X)$ generated by $\widetilde{C}(K)$ and $\left\{S_{t} \psi_{p}, \overline{S_{t} \psi_{p}} ; t \in \mathbf{Q}\right\}$, where $\mathbf{Q}$ is the field of rational numbers. Let $\Sigma$ be the 
maximal ideal space of $C_{0}$. Then $C_{0}$ is isometrically isomorphic to $C(\Sigma)$ by the Gelfand-Naimark theorem. Since $C_{0}$ is invariant under the translations by $\left\{S_{t}\right\}_{t \in \mathbf{R}}$, a one-parameter group of homeomorphisms on $\Sigma$ is induced, and we denote it again by $\left\{S_{t}\right\}_{t \in \mathbf{R}}$. Then the flow $\left(\Sigma,\left\{S_{t}\right\}_{t \in \mathbf{R}}\right)$ is obtained as a factor of $\left(X,\left\{S_{t}\right\}_{t \in \mathbf{R}}\right)$. Indeed, $\Sigma$ is the quotient of $X$ by the relation $\sim$, where $\omega \sim v$ if and only if $\phi(\omega)=\phi(v)$ for all $\phi$ in $C_{0}$. Let $\tau$ be the quotient map of $X$ onto $\Sigma$. Then $\left(\Sigma,\left\{S_{t}\right\}_{t \in \mathbf{R}}\right)$ is a factor of $\left(X,\left\{S_{t}\right\}_{t \in \mathbf{R}}\right)$ under the map $\tau$.

Since $\widetilde{C}(K)$ is a closed subalgebra of $C_{0}$, we see similarly that $\left(\Sigma,\left\{S_{t}\right\}_{t \in \mathbf{R}}\right)$ is an extension of $\left(K,\left\{T_{t}\right\}_{t \in \mathbf{R}}\right)$ under the map

$$
\pi(\omega)=\pi_{p} \circ \tau^{-1}(\omega), \quad \omega \in \Sigma .
$$

We regard $\mu$ as an invariant, ergodic, probability measure in $M(\Sigma)$. Let $H^{p}(\mu)$ be the Hardy space on $\left(\Sigma,\left\{S_{t}\right\}_{t \in \mathbf{R}}\right)$ relative to this measure $\mu$. Then, by regarding $\psi_{p}$ as a function on $\Sigma$, Lemma 2.3 shows that $H_{0}^{p}(\mu)$ is generated by one element $\psi_{p}$.

Let $\Pi$ be the isomorphism of $C(K)$ into $C(X)$ defined by (1.4). Since

$$
\int_{\Sigma} \Pi \phi d \mu=\int_{K} \phi d \sigma, \quad \phi \in C(K)
$$

$\Pi$ extends to an isometry of $L^{p}(\sigma)$ into $L^{p}(\mu)$, naturally. From this, (ii) follows readily.

When $\Gamma$ is countable, the algebra $C_{0}$ is separable. Since $\Sigma$ is compact, this shows that $\Sigma$ is metrizable; thus (iii) holds.

This completes the proof.

\section{Refinements using Helson's Device}

As we mentioned earlier, it is not known whether $H_{0}^{p}(\sigma)$ has a single generator. Using cocycles on $\left(K,\left\{T_{t}\right\}_{t \in \mathbf{R}}\right)$, Helson explored the problem in [9] and [10]. Combining his arguments with our Theorem 1.1, we are able to obtain information on the structure of generators of $H_{0}^{p}(\mu)$.

Let $\left(\Omega,\left\{U_{t}\right\}_{t \in \mathbf{R}}\right)$ be a flow, and let $\nu$ be an invariant, ergodic, probability measure in $M(\Omega)$. A unitary Borel function $A(\omega, t)$ on $\Omega \times \mathbf{R}$ is a (multiplicative) cocycle if $A$ satisfies the cocycle identity

$$
A(\omega, t+s)=A(\omega, t) \cdot A(\omega+t, s), \quad(\omega, s, t) \in \Omega \times \mathbf{R} \times \mathbf{R} .
$$

We identify any two cocycles which differ only on a set of $\nu \times m_{\mathbf{R}}$-measure zero. A cocycle is a (multiplicative) coboundary if it has the form $\phi(\omega) \cdot \overline{\phi(\omega+t)}$ for some unitary function $\phi$ on $K$. If a cocycle is a coboundary multiplied by exp iat for some $\alpha$ in $\mathbf{R}$, then such a cocycle is said to be (multiplicatively) trivial.

Let $u(\omega, t)$ be a real Borel function on $\Omega \times \mathbf{R}$. If $u$ satisfies the identity

$$
u(\omega, t+s)=u(\omega, t)+u(\omega+t, s), \quad(\omega, s, t) \in \Omega \times \mathbf{R} \times \mathbf{R},
$$

then $u$ is called an additive cocycle. When $u$ has the form $v(\omega+t)-v(\omega)$ for some real Borel function $v$ on $\Omega, u$ is called an additive coboundary. We say that $u$ is additively trivial, if $u$ is an additive coboundary plus $\alpha t$ for some $\alpha$ in $\mathbf{R}$.

Let $w_{r}$ be the function in $L_{b u}^{\infty}(\sigma)$ given by (4.1). By using the notation in Section 4 , the function $w_{r} \circ \pi_{p}$ on $X$ satisfies (1.1) for $\mu$-a.e. $\omega \in X$. Then the conjugate function of $t \rightarrow \log w_{r} \circ \pi_{p}(\omega+t)$ becomes an additive cocycle $u(\omega, t)$ on $X \times \mathbf{R}$, by suitable normalization. From the proof Theorem 1.1, we see that there is a single generator $\psi^{(\alpha)}$ of $H_{0}^{p}(X, \mu)$ such that $\left|\psi^{(\alpha)}\right|=\left(w_{r} \circ \pi_{p}\right)^{\alpha}, 0<\alpha \leq 1$. This shows that $\exp i \alpha u(\omega, t)$ is a coboundary for all $\alpha$ in $\mathbf{R}$. 
Nevertheless, as in the proof of [10, Theorem 1], we may show that $u$ cannot be additively trivial, unless $\log w_{r} \circ \pi_{p}$ belongs to $L^{1}(\mu)$. Thus we obtain the following:

Proposition 5.1. In the setting of $L^{p}(\mu)$, the cocycle $\exp i \alpha u(\omega, t)$ on $X \times \mathbf{R}$ is always a coboundary for each $\alpha$ in $\mathbf{R}$, although $u(\omega, t)$ cannot be additively trivial.

This suggests that the theorems in [8] and [12] heavily depend on the separability condition.

Furthermore, [10, Theorem 2] yields that $\left(\Sigma,\left\{S_{t}\right\}_{t \in \mathbf{R}}\right)$ may not be conjugate to $\left(K,\left\{T_{t}\right\}_{t \in \mathbf{R}}\right)$ under ordinary circumstances. Precisely, let $\psi$ be a single generator of $H_{0}^{p}(\mu)$, and let $q=\psi /|\psi|$. We identify $L^{p}(\sigma)$ with a subspace of $L^{p}(\mu)$ by part (ii) of Theorem 1.1. Then the unitary part $q$ of $\psi$ may not usually belong to $L^{p}(\sigma)$, since $|\psi|$ always lies in $L^{p}(\sigma)$ in our construction. We, however, may make the unitary part of a generator lie in $L^{\infty}(\sigma)$.

Proposition 5.2. There is a single generator $\phi$ of $H_{0}^{p}(\mu)$ of which the unitary part $\phi /|\phi|$ lies in $L^{\infty}(\sigma)$.

Proof. Let $\psi$ be a single generator of $H_{0}^{p}(\mu)$ such that $|\psi|$ lies in $L^{p}(\sigma)$. It follows from $\left[9\right.$, Theorem 23] that there is a function $\psi_{1}$ in $H^{p}(\sigma)$ for which $\left|\psi_{1}\right|=|\psi|$. Observe that the unitary function $\vartheta=\psi_{1} / \psi$ lies in $H^{\infty}(\mu)$. If we set

$$
\phi=\psi_{1} \cdot(1+\operatorname{Re} \vartheta),
$$

then $\phi$ satisfies the desired properties.

Incidentally, by virtue of [16], we may describe the structure of $\left(\Sigma,\left\{S_{t}\right\}_{t \in \mathbf{R}}\right)$ in detail. On the other hand, a single generator of $H_{0}^{2}(\mu)$ has a Fourier expansion in the sense of Hilbert space. These facts should be useful to decide whether there can be a single generator in $H_{0}^{2}(\sigma)$. The author conjectures that this should be possible, under suitable assumptions on $\Gamma$.

\section{REMARKS}

(a) Let $\left(\Omega,\left\{U_{t}\right\}_{t \in \mathbf{R}}\right)$ be a minimal flow, meaning that each orbit is dense in $\Omega$. Suppose that $\nu$ is an invariant, ergodic, probability measure in $M(\Omega)$. The argument found in the proof of [6, Chapter VII, Theorem 9.4] works on minimal flows and establishes a weak version of Arens's theorem: If a nonzero function $\phi$ is in $A(\Omega)$, then $\log |\phi|$ lies in $L^{1}(\nu)$.

Let $\left(\Sigma,\left\{S_{t}\right\}_{t \in \mathbf{R}}\right)$ and $\mu$ be as in Theorem 1.1. Then $\left(\Sigma,\left\{S_{t}\right\}_{t \in \mathbf{R}}\right)$ cannot be minimal, because the single generator $\psi_{p}$ of $H_{0}^{p}(\mu)$ in the proof is continuous on $\Sigma$, while $\log \left|\psi_{p}\right|$ is not summable by Szegö's theorem.

(b) Suppose that $\Gamma$ is countable. By part (iii) of Theorem 1.1, the measure space $(\Sigma, \mu)$ is a standard Borel probability space. Then [2] asserts that the ergodic flow defined by $\left(\Sigma,\left\{S_{t}\right\}_{t \in \mathbf{R}}\right)$ and $\mu$ is conjugate to a strictly ergodic flow $\left(Y,\left\{V_{t}\right\}_{t \in \mathbf{R}}\right)$. Thus we obtain the following:

Proposition 6.1. There is a strictly ergodic flow $\left(Y,\left\{V_{t}\right\}_{t \in \mathbf{R}}\right)$ on which $H_{0}^{p}(\nu)$, $1 \leq p \leq \infty$, has a single generator, where $\nu$ is the unique invariant probability measure in $M(Y)$.

This enables us to strengthen the conclusion of Theorem 1.1 substantially. Since $\Gamma$ contains $2 \pi$, we may assume that a continuous eigenfunction with eigenvalue $2 \pi$ exists on $\left(Y,\left\{V_{t}\right\}_{t \in \mathbf{R}}\right)$. This implies that $\left(Y,\left\{V_{t}\right\}_{t \in \mathbf{R}}\right)$ is built from a discrete flow. 
So a repetition of the argument in Section 3 leads to the fact that $\left(X,\left\{S_{t}\right\}_{t \in \mathbf{R}}\right)$ is also an extension of $\left(Y,\left\{V_{t}\right\}_{t \in \mathbf{R}}\right)$ under a continuous map $\pi$ of $X$ onto $Y$.

Proposition 6.2. Let $\left(X,\left\{S_{t}\right\}_{t \in \mathbf{R}}\right)$ be the flow introduced in Section 2, and let $\lambda$ be any invariant, ergodic, probability measure in $M(X)$. Then $H_{0}^{p}(\lambda)$ has a single generator.

Proof. Since $\left(Y,\left\{V_{t}\right\}_{t \in \mathbf{R}}\right)$ is strictly ergodic, we see that

$$
\int_{X} \phi \circ \pi d \lambda=\int_{Y} \phi d \nu, \quad \phi \in C(Y)
$$

Then the map $\phi \rightarrow \phi \circ \pi$ induces an isometry of $L^{p}(\nu)$ into $L^{p}(\lambda)$. By this isometry, we may regard $H_{0}^{p}(\nu)$ as a subspace of $H_{0}^{p}(\lambda)$. We recall that $H_{0}^{p}(\nu)$ has a single generator. Therefore, it follows from Lemma 2.3 that $H_{0}^{p}(\lambda)$ is singly generated.

\section{REFERENCES}

1. R. Curto, P. Muhly, and T. Nakazi, Ergodic $H^{1}$ is not a dual space, Hokkaido Math. J. 16 (1987), 75-79. MR 88f:46084

2. M. Denker and E. Eberlein, Ergodic flows are strictly ergodic, Adv. in Math. 13 (1974), 437-473. MR 50:4890

3. N. Dunford and J. Schwartz, Linear operators, Part I, Wiley Interscience, New York, 1958. MR 22:8302

4. F. Forelli, Analytic and quasi-invariant measures, Acta Math. 118 (1967), 33-59. MR 35:667

5. T. Gamelin, $H^{p}$ spaces and extremal functions in $H^{1}$, Trans. Amer. Math. Soc. 124 (1966), 158-167. MR 35:4731

6. — Uniform algebras, Prentice-Hall, Englewood Cliffs, NJ, 1969. MR 53:14137

7. J. Garnett, Bounded analytic functions, Academic Press, New York, 1981. MR 83g:30037

8. T. Hamachi, Y. Oka, and M. Osikawa, A classification of ergodic nonsingular transformation groups, Mem. Fac. Sci. Kyushu Univ. Ser. A 28 (1974), 113-133. MR 55:5839

9. H. Helson, Analyticity on compact abelian groups, Algebras in Analysis, Academic Press, London, 1975, pp. 1-62. MR 55:989

10. _ Compact groups with ordered duals. VII, J. London Math. Soc. (2) 20 (1979), 509515. MR 81m:43013

11. _ The spectral theorem, Lecture Notes in Math., vol. 1227, Springer-Verlag, Berlin, 1986. MR 88k: 47027

12. C. C. Moore and K. Schmidt, Coboundaries and homeomorphisms for nonsingular actions and a problem of H. Helson, Proc. London Math. Soc. (3) 40 (1980), 443-475. MR 82a:22007

13. P. Muhly, Function algebras and flows, Acta Sci. Math. (Szeged) 35 (1973), 111-121. MR 48:9403a

14. _ Function algebras and flows, IV, Trans. Amer. Math. Soc. 203 (1975), 55-66. MR 58:12383b

15. Isometries of ergodic Hardy spaces, Israel J. Math. 36 (1980), 50-74. MR 82d:28018

16. W. Parry, Compact abelian group extensions of discrete dynamical systems, Z. Wahrsch. Verw. Gebiete 13 (1969), 95-113. MR 41:5596

17. J. Tanaka, Blaschke cocycles and generators, Pacific J. Math. 142 (1990), 357-378. MR 91f: 22006

18. , Flows in fibers, Trans. Amer. Math. Soc. 343 (1994), 779-804. MR 94h:46081

19. T. Srinivasan and J. Wang, Weak-* Dirichlet algebras, Function Algebras, Scott-Foresman, Chicago, 1966, pp. 216-249. MR 33:6441

20. R. Walker, The Stone-Čech compactification, Springer-Verlag, Berlin, 1974. MR 52:1595

Department of Mathematics, Tsuru University, Tsuru City, Yamanashi 402, Japan

E-mail address: tanaka@tsuru.ac.jp 